\title{
Connexin and Pannexin-Based Channels in Oligodendrocytes: Implications in Brain Health and Disease
}

\author{
Sebastián Vejar ${ }^{1}$, Juan E. Oyarzún ${ }^{2,3}$, Mauricio A. Retamal ${ }^{4,5 *}$, Fernando C. Ortiz ${ }^{1 *}$, \\ and Juan A. Orellana ${ }^{2,3 *}$

\begin{abstract}
'Mechanisms of Myelin Formation and Repair Laboratory, Instituto de Ciencias Biomédicas, Facultad de Ciencias de la Salud, Universidad Autónoma de Chile, Santiago, Chile, ${ }^{2}$ Departamento de Neurología, Escuela de Medicina and Centro Interdisciplinario de Neurociencias, Facultad de Medicina, Pontificia Universidad Católica de Chile, Santiago, Chile, ${ }^{3}$ Centro de Investigación y Estudio del Consumo de Alcohol en Adolescentes, Pontificia Universidad Católica de Chile, Santiago, Chile, ${ }^{4}$ Centro de Fisiología Celular e Integrativa, Facultad de Medicina, Clínica Alemana Universidad del Desarrollo, Santiago, Chile, ${ }^{5}$ Department of Cell Physiology and Molecular Biophysics, and Center for Membrane Protein Research, Texas Tech University Health Sciences Center, Lubbock, TX, United States
\end{abstract}

OPEN ACCESS

Edited by:

Francesco Moccia, University of Pavia, Italy

Reviewed by:

Bela Volgyi,

University of Pécs, Hungary

Randy Franklin Stout, New York Institute of Technology, United States

${ }^{*}$ Correspondence:

Mauricio A. Retamal mretamal@udd.cl Fernando C. Ortiz fernando.ortiz@uautonoma.cl Juan A. Orellana jaorella@uc.cl

Received: 26 October 2018 Accepted: 07 January 2019 Published: 29 January 2019

Citation: Vejar S, Oyarzún JE, Retamal MA, Ortiz FC and Orellana JA (2019) Connexin and Pannexin-Based Channels in Oligodendrocytes: Implications in

Brain Health and Disease.

Front. Cell. Neurosci. 13:3. doi: 10.3389/fncel.2019.00003
Oligodendrocytes are the myelin forming cells in the central nervous system (CNS). In addition to this main physiological function, these cells play key roles by providing energy substrates to neurons as well as information required to sustain proper synaptic transmission and plasticity at the CNS. The latter requires a fine coordinated intercellular communication with neurons and other glial cell types, including astrocytes. In mammals, tissue synchronization is mainly mediated by connexins and pannexins, two protein families that underpin the communication among neighboring cells through the formation of different plasma membrane channels. At one end, gap junction channels (GJCs; which are exclusively formed by connexins in vertebrates) connect the cytoplasm of contacting cells allowing electrical and metabolic coupling. At the other end, hemichannels and pannexons (which are formed by connexins and pannexins, respectively) communicate the intra- and extracellular compartments, serving as diffusion pathways of ions and small molecules. Here, we briefly review the current knowledge about the expression and function of hemichannels, pannexons and GJCs in oligodendrocytes, as well as the evidence regarding the possible role of these channels in metabolic and synaptic functions at the CNS. In particular, we focus on oligodendrocyte-astrocyte coupling during axon metabolic support and its implications in brain health and disease.

Keywords: connexons, oligodendrocytes, pannexons, hemichannels, gap junctions, demyelinating neuropathy

\section{INTRODUCTION}

Oligodendrocytes are the myelin-forming cells of the central nervous system (CNS). They were first described and characterized as small cells with many branches distributed ubiquitously within the CNS in a close spatial relationship with axons (del Río-Hortega, 1922; Ramón y Cajal, 1925). Oligodendrocyte processes wrap the axon at different points, forming the multilamellar 
structure of myelin, which is organized in sheaths along the axonal axis. Myelin sheaths enable axons for saltatory conduction accounting for the speed-up of action potential conduction in neurons (Tasaki, 1939; Nave and Werner, 2014). By exerting this main function, oligodendrocytes largely contribute to establish the conduction properties of neural circuits, leading to the proper integration of electrical signaling at the CNS. Consequently, in diseases characterized by the loss of myelin-such as multiple sclerosis (MS) - patients show several neurological impairments, revealing the decisive contribution of oligodendrocytes to brain function (Franklin and FfrenchConstant, 2008, 2017).

In addition to their key role on saltatory conduction, oligodendrocytes participate in other important functions ranging from metabolic supply and ion buffering to setting survival and excitability properties of neurons (Menichella et al., 2006; Hamada and Kole, 2015; Battefeld et al., 2016; Saab et al., 2016; Philips and Rothstein, 2017). The plastic nature of oligodendrocytes relies not only in their ability to fulfill diverse functions, but also on the fact that they conform heterogeneous populations with different properties. Indeed, according to their morphological complexity, size and distribution, oligodendrocytes can be grouped into four subtypes (del Río-Hortega, 1922; Pérez-Cerdá et al., 2015). Type I and II represent the less abundant population, which myelinates mainly small axons in the corpus callosum and the cortex, while type III and IV are associated to the myelination of larger axons in the spinal cord (Dimou and Simons, 2017). A different grouping criterion-based mainly in the oligodendrocyte location around neurons-identify another subtype of oligodendrocytes, named satellite oligodendrocytes (Ludwin, 1979; Takasaki et al., 2010; Battefeld et al., 2016). Satellite oligodendrocytes are normally located at perineural gray matter regions, and although they have not been extensively characterized, evidence indicates that this population could play also trophic roles by giving metabolic support and extracellular $\mathrm{K}^{+}$buffering to neurons (Szuchet et al., 2011; Battefeld et al., 2016). Most of the current literature characterize satellite oligodendrocyte as a non-myelinating population, however, recent studies have shown that under certain conditions (i.e., demyelination) they actually synthetize myelin, forcing to revisit the current notion of satellite oligodendrocytes usually based on their inability to synthetize myelin (Szuchet et al., 2011; Battefeld et al., 2016).

In the nervous system, oligodendrocytes interact with several neighboring cells by multiple mechanisms. For instance, they sense growth factors and cytokines released by surrounding astrocytes and microglial cells (Ishibashi et al., 2006; Miron et al., 2013), particularly, under pathological conditions, where these signals can induce the maturation of oligodendrocyte precursor cells (OPCs) into oligodendrocytes or stabilize their mature phenotype (Fulmer et al., 2014; Hammond et al., 2015; Miyamoto et al., 2015). Similarly, oligodendrocytes and OPCs can respond to neural activity by detecting neurotransmitters through the activation of ionotropic and metabotropic receptors (i.e., AMPARs, NMDAR, mGluRs,
GABAxRs and nAChRs; Bergles et al., 2000; Lin and Bergles, 2004; Kukley et al., 2007; Vélez-Fort et al., 2009; Li et al., 2013; Orduz et al., 2015; Wake et al., 2015; Saab et al., 2016; Fields et al., 2017). Commonly, glial cells communicate each other and with neurons via the release of bioactive molecules called gliotransmitters (Araque et al., 2014). Although it is unclear whether oligodendrocytes are endowed with the machinery to allow the release of gliotransmitters, they form extensive functional interactions among them and with astrocytes through special structures called gap junctions (GJs).

GJs result from the accumulation of intercellular channels in areas of close apposition between two plasma membranes of adjacent cells. These channel-called GJs channels (GJCs)favor the direct intercellular exchange of metabolites (e.g., ADP, glucose, glutamate and glutathione), second messengers (e.g., cAMP and $\mathrm{IP}_{3}$ ) and ions, allowing the cell-cell spread of electrotonic potentials in excitable and non-excitable tissues (Leybaert et al., 2017). When two hemichannels from adjacent cells align with each other in appositional membranes, they make a full intercellular GJC (Saez et al., 2003). Each hemichannel is constituted by the oligomerization of six monomers of connexins, a highly conserved protein family encoded by 21 genes in humans and 20 in mice, with orthologs in other vertebrate species (Abascal and Zardoya, 2013). Recent data indicates that along with constitute the building blocks of GJCs, hemichannels in nonjunctional membranes may serve as diffusional routes for ion and small molecules between the cytoplasm and the extracellular compartment (Montero and Orellana, 2015). Fifteen years ago, a new gene family encoding a set of three membrane proteins termed pannexins was discovered (Bruzzone et al., 2003). Despite that pannexins do not share significant amino acid sequences with connexins, both families have analogous secondary and tertiary structures (Abascal and Zardoya, 2013). Most of findings implicate that pannexins are capable to form single membrane channels, termed pannexons, that have some similarities with hemichannels (Sosinsky et al., 2011). Under healthy conditions, hemichannels and pannexons underpin the release of gliotransmitters (e.g., ATP, glutamate, D-serine, lactate), acting as crucial players in multiple brain processes such as synaptic transmission, neuronal oscillations, glucose sensing, ischemic tolerance and fear memory consolidation (Lin et al., 2008; Orellana et al., 2012; Stehberg et al., 2012; Chever et al., 2014; Roux et al., 2015; Meunier et al., 2017). Nonetheless, the permanent and exacerbated activity of these channels might operate as a cornerstone in the prelude and development of homeostatic imbalances detected in diverse neuropathological diseases (Orellana et al., 2016).

In this work, we review and discuss the evidence sustaining the possible role of oligodendrocyte GJ coupling in the coordination of metabolic support for neuronal activity, as well as its participation in demyelination processes observed in different diseases. In addition, we overview the recent evidence arguing for the functional expression of hemichannels and pannexons in oligodendrocytes and OPCs and how this may impact not only their normal 
metabolism, but also their survival during inflammatory scenarios.

\section{OLIGODENDROCYTE- OLIGODENDROCYTE GAP JUNCTIONAL COMMUNICATION AND THEIR CONTRIBUTION TO HEALTH AND DISEASE}

\section{General Characteristics of Connexin Expression in Oligodendrocytes}

Oligodendrocytes have been reported to express connexin 29 (Cx29), Cx32, Cx45 and Cx47 (Figure 1; Dermietzel et al., 1989; Micevych and Abelson, 1991; Nagy et al., 2003; Li et al., 2004). Although the latter could imply that any of these connexins may form GJCs in oligodendrocytes, early studies showed contradictory experimental data, revealing the complexity of detecting GJ structures in these cells (OrthmannMurphy et al., 2008; Nualart-Marti et al., 2013). Pioneering studies using freeze-fracture electron microscopy (FEM) in rat embryonic cultures described that contrary to that found in astrocytes, oligodendrocytes do not form GJCs between them (Massa and Mugnaini, 1982). Comparable results were obtained from FEM experiments in adult cat brain and spinal cord, were astrocyte-astrocyte and astrocyte-oligodendrocyte GJs were observed, but inter-oligodendrocyte GJs were not detected (Massa and Mugnaini, 1982). Similarly, freeze-fracture of freshly isolated oligodendrocytes from adult lamb brains, showed that GJs between oligodendrocytes were small and infrequent (Massa et al., 1984).

Contrasting the above findings, Kettenmann et al. (1983) described the successful coupling between oligodendrocytes from 2-week old mouse explants, as measured by dye transfer and electrophysiological recordings. An interesting result of this work was that about $34 \%$ of oligodendrocytes were electrically coupled, whereas the $70 \%$ exhibited positive Lucifer yellow diffusion to another oligodendrocyte. Indeed, dye-coupled oligodendrocytes were seen as far as $300 \mu \mathrm{m}$ from the original injected cell (Kettenmann et al., 1983). This study was further supported by findings indicating that long term cultured oligodendrocytes from adult bovines establish GJ-like structures between them, as observed through electron microscopy (Norton et al., 1983). The apparent contradiction in which early studies described virtually no GJ-like structures in oligodendrocytes vs. other findings showing the opposite, probably relies on technical discrepancies, as well as the complex diversity of oligodendrocytes and their differential pattern of connexin expression. For instance, oligodendrocytes expressing $\mathrm{O} 1$ or $\mathrm{O} 4$ do not display dye or electrical coupling, while those expressing $\mathrm{O} 10$ show almost $40 \%$ coupling (Von Blankenfeld et al., 1993). Additionally, in slices from rat spinal cords, about $18 \%$ of oligodendrocytes in the gray matter are coupled, as showed by Lucifer yellow and neurobiotin coupling assays, whereas oligodendrocyte from white matter are not (Pastor et al., 1998). The latter findings suggest that oligodendrocyte gap junctional communication may change depending on the CNS area. Nowadays, the accumulating in vivo and in vitro evidence indicates that oligodendrocytes are coupled to each other through homotypic GJCs formed mainly by $\mathrm{Cx} 47$ and secondarily by $\mathrm{Cx} 32$ (Figures 1, 2; Maglione et al., 2010; Wasseff and Scherer, 2011).

Myelin sheaths of both Schwann cells and oligodendrocytes are intraconnected through GJs formed by $\mathrm{Cx} 32$, allowing the flux of ions and molecules from the perinuclear cytoplasm to the rest of the cell (Balice-Gordon et al., 1998; Nagy et al., 2003; Kamasawa et al., 2005). In fact, cultured oligodendrocytes form tight junctions and GJCs between their myelin sheaths, reassembling what occur in vivo (Gonatas et al., 1982). Although myelin sheaths express Cx29 (Cx31.3 in humans; Sargiannidou et al., 2008) and Cx32 (Nagy et al., 2003; Li et al., 2004; Nualart-Marti et al., 2013), they do not colocalize with each other. Particularly, Cx29 is detected in the inner/adaxonal membrane (opposing the axonal membrane) of small myelinated fibers (Altevogt et al., 2002), whereas Cx32 is localized from the soma towards the abaxonal membrane of primarily large myelinated sheaths and among contiguous layers of myelin membrane at the paranode (Kleopa et al., 2004; Kamasawa et al., 2005). Of note, despite its localization in the cell body of oligodendrocytes, Cx29 seems to form hemichannels, likely in the adaxonal membrane, rather than function as GJC (Altevogt et al., 2002; Altevogt and Paul, 2004; Kamasawa et al., 2005; Ahn et al., 2008; Orthmann-Murphy et al., 2008). In agreement with this idea, Cx31.3-the human ortholog of murine Cx29exhibit functional features compatible with the formation of hemichannels, but not GJCs (Sargiannidou et al., 2008). In the white and gray matter, $\mathrm{Cx} 47$ localizes in the cell body and initial processes of oligodendrocytes, as well as in the abaxonal membrane of myelinated fibers (Nagy et al., 2003; Altevogt and Paul, 2004; Kleopa et al., 2004; Kamasawa et al., 2005). In those cell areas Cx47 may form homotypic Cx47/Cx47 GJCs among oligodendrocytes and heterotypic GJCs with astrocytes expressing Cx43 (see next section; Figure 2; Wasseff and Scherer, 2011; Fasciani et al., 2018).

\section{Functional and Dysfunctional Coupling Among Oligodendrocytes}

Astrocytes are extensively coupled to each other through homotypic GJCs composed by Cx30 or Cx43 (Giaume et al., 1991; Nagy et al., 2001). For a long time, they were considered as the major protagonists in the buffering of extracellular $\mathrm{K}^{+}$from the synaptic cleft (Walz, 2000). Nonetheless, recent evidence indicates that oligodendrocytes along with astrocytes may form a "panglial syncytium" connected through GJs, constituting the principal pathway for the long-distance siphoning of $\mathrm{K}^{+}$from the juxtaparanodal periaxonal space to vasculature or the cerebrospinal fluid (Menichella et al., 2006; Rash, 2010). Oligodendrocytes form heterocellular GJs with astrocytes through heterotypic channels of $\mathrm{Cx} 47$ with $\mathrm{Cx} 43$, but also by Cx32/Cx30 GJCs depending on the anatomical area (Figure 2; Orthmann-Murphy et al., 2007b; Maglione et al., 2010; Magnotti et al., 2011; Wasseff and Scherer, 2011; Tress et al., 2012; Fasciani et al., 2018). This unequal connexin combination of GJCs could derive in a unidirectional rather than a bidirectional ion and molecular interchange between oligodendrocyte and astrocytes 


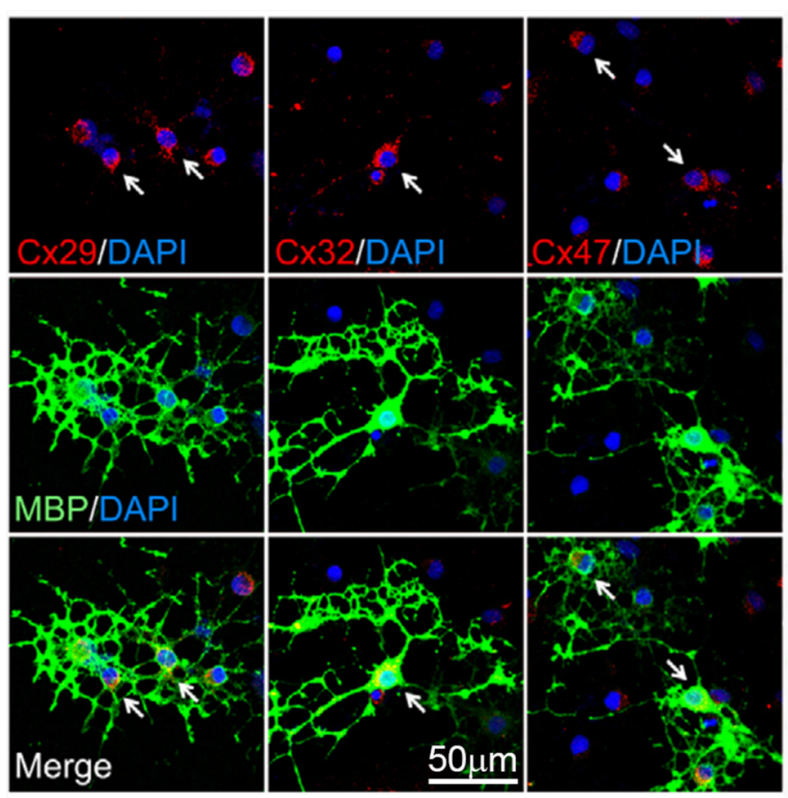

FIGURE 1 | Expression of connexins by oligodendrocytes. Representative fluorescence images depicting the double immunostaining of connexin 29 (Cx29; red, left panels), Cx32 (red, middle panels) and Cx47 (red, right panels) with the oligodendrocyte-specific bio-marker myelin basic protein (MBP, green) and DAPI staining (blue) by mature cultured oligodendrocytes after 6 days in vitro. Arrows highlight representative MBP-positive cells. Adapted, with permission, from Niu et al. (2016).

(Flagg-Newton and Loewenstein, 1980). This may imply the existence of a directional diffusion barrier that might account for the spatial buffering of periaxonal $\mathrm{K}^{+}$as well as for metabolic coupling and signaling between these cell types. Consistent with this notion, $\mathrm{Cx} 32 / \mathrm{Cx} 30$ GJCs show an evident fast ionic rectification depending on the polarity of transjunctional voltage (Orthmann-Murphy et al., 2007b), whereas Cx47/Cx43 GJCs display ionic and chemical rectification, which result in a directional permeability barrier for the movement of ions and molecules from cells expressing $\mathrm{Cx} 47$ to those endowed with Cx43 (Fasciani et al., 2018). Interestingly, electron microscopy and freeze-fracture replica immunogold labeling revealed that voltage-gated $\mathrm{K}^{+}$channels Kv1.1/Kv1.2 form densely packed "rosettes" that are exactly aligned with Cx29 hemichannels in the surrounding juxtaparanode myelin collars and along the inner mesaxon (Rash et al., 2016). Whether Cx29 functionally docks with $\mathrm{Kv1.1/Kv1.2} \mathrm{to} \mathrm{constitute} \mathrm{a} \mathrm{full} \mathrm{myelin-axon} \mathrm{channel}$ involved in $\mathrm{K}^{+}$buffering remains to be elucidated. If the existence of this new entity is confirmed, it will open a new line of the study in the field, as the discover of pannexins did years ago.

An additional key function of oligodendrocytes is their ability to provide metabolic support to the axonal compartment of neurons (Saab et al., 2016; Philips and Rothstein, 2017; Meyer et al., 2018). Although the underlying mechanisms of this phenomenon are not completely understood, GJCs between oligodendrocytes and astrocytes, as well as direct oligodendrocyte-neuron gap junctional communication have been pointed out as major mediators on this process (Nualart-
Marti et al., 2013; Philips and Rothstein, 2017; Meyer et al., 2018). A recent study, from Niu et al. (2016) attempted to evaluate whether connexin-based channels contribute to glucose trafficking pathways in oligodendroglial cells. They observed that a glucose analog can diffuse through oligodendrocyte-astrocyte but not OPC-astrocyte GJs, suggesting the presence of a panglial metabolic route that may depend on the developmental stage of the oligodendroglial lineage (Niu et al., 2016). In agreement with these data, cells expressing the surface ganglioside A2B5 and OPCs are not dye or electrically coupled to astrocytes whereas mature oligodendrocytes do couple with astrocytes (Bergles et al., 2000; Lin and Bergles, 2004; Xu et al., 2014). Moreover, oligodendrocytes have the ability to supply lactate or glucose to maintain axonal function, depending on the white matter region (Saab et al., 2016; Meyer et al., 2018). Interestingly, experiments carried out on the corpus callosum found that in knockout animals for $\mathrm{Cx} 47$, oligodendrocytes lose the ability to maintain axonal function (Meyer et al., 2018). Moreover, this effect was astrocyte independent, suggesting that proper axonal function requires a direct energy supply from oligodendrocytes by a mechanism that may involve the opening of $\mathrm{Cx} 47$-based channels (Figure 2; Meyer et al., 2018). Accordingly, major modifications on GJ-based connectivity have been reported for both oligodendrocytes and astrocytes under demyelinated conditions, where an impaired metabolism is expected (Kleopa et al., 2010; Markoullis et al., 2012, 2014).

How does the disruption of GJ communication among oligodendrocytes contribute to CNS diseases? Charcot-MarieTooth (CMT) disease is importantly correlated with mutations in Cx32 gene (Fairweather et al., 1994; Kleopa et al., 2010; Table 1). This neuropathology primarily affects to Schwann cells, resulting in progressive demyelination (Nelis et al., 1996). Interestingly, oligodendrocytes are much less affected by CMT disease. Indeed, knockout mice for Cx32 show progressive loss of myelin in Schwann cells but not in oligodendrocytes (Scherer et al., 1998). This evidence may imply that $\mathrm{Cx} 32$ does not participate in the formation or maintaining of myelin in oligodendrocytes. Alternatively, the latter may reflect that the role of Cx32 in myelin can be greatly bypassed by other connexins expressed in oligodendrocytes (e.g., Cx47). In agreement with these ideas, it has been suggested that Cx45 expression in oligodendrocytes could prevent the demyelination induced by CMT disease (Kunzelmann et al., 1997). In spite of that, the impaired activity observed in neocortical neurons from the Cx32 knockout mouse likely relies on the decreased thickness of the axonal myelin sheaths (Sutor et al., 2000). Therefore, although the involvement of Cx32 in myelin formation is not as evident as in the peripheral nervous system, subclinical manifestations often can be observed (Sutor et al., 2000; Kleopa et al., 2002).

The role of oligodendrocyte gap junctional communication in demyelinating diseases is still a matter of ongoing research. Mutations in $\mathrm{Cx} 47$ have been found in patients with the central hypomyelinating disorder known as Pelizaeus-Merzbacher-like disease (also known as hypomyelinating leukodystrophy 2) and the hereditary spastic paraplegia (Sargiannidou et al., 2010; Tress et al., 2011; Cotrina and Nedergaard, 2012). In this context, mice bearing a human $\mathrm{Cx} 47^{\mathrm{M} 283 \mathrm{~T}}$ missense mutation 
TABLE 1 | Brief summary of the involvement of connexins and pannexins in demyelinating diseases.

\begin{tabular}{|c|c|c|c|}
\hline Disease & Cell type & Connexin or Pannexin & References \\
\hline \multirow[t]{4}{*}{ Charcot-Marie-Tooth (CMT) } & ND & $\mathrm{C} \times 32$ & $\begin{array}{l}\text { Fairweather et al. (1994), Nelis et al. } \\
\text { (1996) }\end{array}$ \\
\hline & Oligodendrocytes & Cx32 & Olympiou et al. (2016) \\
\hline & Schwann cells and oligodendrocytes & Cx32 & Scherer et al. (1998), Kleopa et al. (2002) \\
\hline & Oligodendrocytes & $\mathrm{Cx} 32$ and $\mathrm{Cx} 45$ & $\begin{array}{l}\text { Kunzelmann et al. (1997), Kleopa et al. } \\
\text { (2010) }\end{array}$ \\
\hline Myelination defects and neuronal hyperexcitability & Schwann cells & $\mathrm{C} \times 32$ & Sutor et al. (2000) \\
\hline $\begin{array}{l}\text { Pelizaeus-Merzbacher-like (hypomyelinating } \\
\text { leukodystrophy 2) and hereditary spastic paraplegia }\end{array}$ & Oligodendrocytes & $\mathrm{C} \times 47$ & $\begin{array}{l}\text { Osaka et al. (2010), Sargiannidou et al. } \\
\text { (2010), Tress et al. (2011), Cotrina and } \\
\text { Nedergaard (2012), Zlomuzica et al. } \\
\text { (2012) }\end{array}$ \\
\hline Oligodendrocyte identity and survival & Oligodendrocytes & Cx47 & $\begin{array}{l}\text { Schlierf et al. (2006), Pozniak et al. } \\
\text { (2010), Takada et al. (2010), Suzuki et al. } \\
\text { (2017) }\end{array}$ \\
\hline Leukodystrophy & Oligodendrocytes Astrocytes & Cx47 & Fasciani et al. (2018) \\
\hline Multiple sclerosis & Oligodendrocytes & $\mathrm{C} \times 32$ and $\mathrm{C} \times 47$ & Markoullis et al. $(2012,2014)$ \\
\hline $\begin{array}{l}\text { Experimental autoimmune encephalomyelitis (model } \\
\text { of multiple sclerosis) }\end{array}$ & Oligodendrocytes & $\mathrm{C} \times 32$ and $\mathrm{Cx} 47$ & Constantinescu et al. (2011) \\
\hline Hypomyelinated leukodystrophy & Oligodendrocytes & $\mathrm{C} \times 32$ and $\mathrm{C} \times 47$ & Wasseff and Scherer (2015) \\
\hline Demyelination & Oligodendrocytes & Panx1 & Hainz et al. (2017) \\
\hline
\end{tabular}

*This table was intended to show several examples and does not correspond to a compilation of all published evidence. ND, not determined.

exhibit a complex age-dependent behavioral phenotype including changes in psychomotor, emotional and memory functions (Zlomuzica et al., 2012). Although with different localizations, both $\mathrm{Cx} 32$ and $\mathrm{Cx} 47$ are transcriptionally regulated by the same transcription factor: Sox10 (Schlierf et al., 2006), determinant in conferring oligodendrocyte identity and its survival (Pozniak et al., 2010; Takada et al., 2010; Suzuki et al., 2017). Moreover, mutations in the Cx47 gene promoter that binds Sox10 are responsible for the demyelination observed in Pelizaeus-Merzbacher-like disease (Osaka et al., 2010). Of particular interest is the absence of $\mathrm{Cx} 47$ in the myelin sheath of oligodendrocytes despite its wide presence in the perikarya (Kleopa et al., 2004; OrthmannMurphy et al., 2007a), where it form heterotypic GJCs with the Cx43 expressed in astrocytes (Kamasawa et al., 2005). Supporting this notion, when $\mathrm{Cx} 43$ is expressed in astrocytes it induces the phosphorylation and further stabilization of $\mathrm{Cx} 47$ (May et al., 2013). Similarly, the restrictive permeability of $\mathrm{Cx} 47 / \mathrm{Cx} 43$ GJCs is suppressed by a mutation $\left(\mathrm{Cx} 47^{\mathrm{P} 90 \mathrm{~S}}\right)$ linked with leukodystrophy (Fasciani et al., 2018), indicating a new pathogenic mechanism underlying myelin disorders that implies impairments in the oligodendrocyte-astrocyte coupling. Thus, the GJ-mediated crosstalk between these glial cells could be more important than originally thought and deserve further investigation.

Data from animal studies indicates that failures in GJC-mediated coupling may contribute to demyelination (Kleopa et al., 2010; Markoullis et al., 2012, 2014). For instance, a murine model of MS-the experimental autoimmune encephalomyelitis (EAE; Constantinescu et al. (2011))-showed a reduction in GJ plaques composed by both $\mathrm{Cx} 32$ or $\mathrm{Cx} 47$ in oligodendrocytes within and around lesions but also in the normal appearing white matter (Markoullis et al., 2012). Of note, the latter was paralleled with decreased numbers of Cx43 GJ plaques in astrocytes. A similar loss of GJs formed by $\mathrm{Cx} 32$ or $\mathrm{Cx} 47$ is observed in oligodendrocytes in the gray matter of post-mortem tissue from MS patients (Markoullis et al., 2014). Instead of what is found in the EAE model, these findings were paralleled with an increase in $\mathrm{C} \times 30$ and $\mathrm{Cx} 43$ along with an augment of astrocytic GJ plaques, indicating a different molecular profile of astrogliosis in white and gray matter pathology associated to MS. Supporting the role of oligodendrocyte connexins in myelination, ablation of $\mathrm{Cx} 32$ and Cx47 generates a phenotype of hypomyelinated leukodystrophy characterized by alterations in gene expression of key enzymes required for the synthesis of myelin lipids, as well as increased expression of genes linked with leukotrienes/prostaglandins synthesis and chemokines/cytokines interactions and signaling pathways (Wasseff and Scherer, 2015). The latter suggests that loss of oligodendrocyte-oligodendrocyte and oligodendrocyteastrocyte coupling is accompanied by a prominent immune response. Likewise, the knockout mice for $\mathrm{C} \times 32$ have a greater sensitivity to inflammatory challenges and cellular stressors (Olympiou et al., 2016), arguing for the relevance of oligodendrocytes GJ signaling in the inflammatory and immune response. Although these findings strongly suggest a role for oligodendrocyte and/or oligodendrocyte-astrocyte coupling in the pathogenesis and progression of demyelinated lesions (see Table 1), functional approaches are necessary to draw definitive conclusions and uncover the underlying mechanisms.

\section{HEMICHANNELS AND PANNEXONS IN OLIGODENDROCYTES}

Despite that hemichannels and pannexons were discovered almost three and two decades ago, respectively (Paul et al., 1991; Bruzzone et al., 2003), the available evidence showing their functional expression in oligodendrocytes is still limited and relatively recent. Pioneering work by Niu et al. (2016) 
showed for first time the presence of functional hemichannels in oligodendrocytes. Using in vitro primary cell cultures, they demonstrated that the opening of hemichannels mediates the uptake of a glucose analog in OPCs and oligodendrocytes, this response being more prominent in the former and highly dependent on $\left[\mathrm{Ca}^{2+}\right]_{\mathrm{i}}$ (Niu et al., 2016). Importantly, the inhibition of these channels strongly reduced OPC proliferation, suggesting that the uptake of glucose via hemichannels is critical for the development of the oligodendroglia lineage. More relevant, when oligodendrocytes are co-cultured with astrocytes knocked-out for $\mathrm{Cx} 43$, a decrease in extracellular glucose was found paralleled with a reduction in OPC proliferation (Niu et al., 2016). Therefore, in OPCs, hemichannels may provide a major pathway for glucose entry, which could be supplemented by $\mathrm{Cx} 43$-mediated coupling between astrocytes and oligodendrocytes and/or the release of glucose or its metabolites (e.g., lactate) through astrocyte Cx43 hemichannels.

A recent study showed that prenatal exposure to high levels of glucocorticoids increases the dye uptake of oligodendrocytes in brain slices of the offspring (Maturana et al., 2017). This response was eliminated by the application of Gap26, a mimetic peptide that in short periods of exposure (1-15 min) specifically inhibits $\mathrm{Cx} 43$ hemichannels rather than $\mathrm{Cx} 43$ GJCs. Similar effects were found with A740003, a selective $\mathrm{P}_{2} \mathrm{X}_{7}$ receptor $\left(\mathrm{P} 2 \mathrm{X}_{7} \mathrm{R}\right)$ antagonist, and with the mimetic peptide ${ }^{10}$ panx1, which block Panx1 channels. Given that oligodendrocytes do not express $\mathrm{Cx} 43$, the authors explained these results by proposing that high levels of glucocorticoids could increase the activity of Cx43 hemichannels in microglia or astrocytes, consequently increasing the extracellular levels of ATP and positively impacting the opening of Panx1 channels in oligodendrocytes following the stimulation of $\mathrm{P}_{2} \mathrm{X}_{7} \mathrm{Rs}$. In agreement with this idea, the glucocorticoid-induced dye uptake by oligodendrocytes depended on the activation of mast cells and microglia and was accompanied of increased expression of major elements of the inflammasome, including NLRP3, ASC and caspase-1 (Maturana et al., 2017). Despite the above, whether this phenomenon is the result of the upstream activation of $\mathrm{Cx} 43$ hemichannels in other brain cells

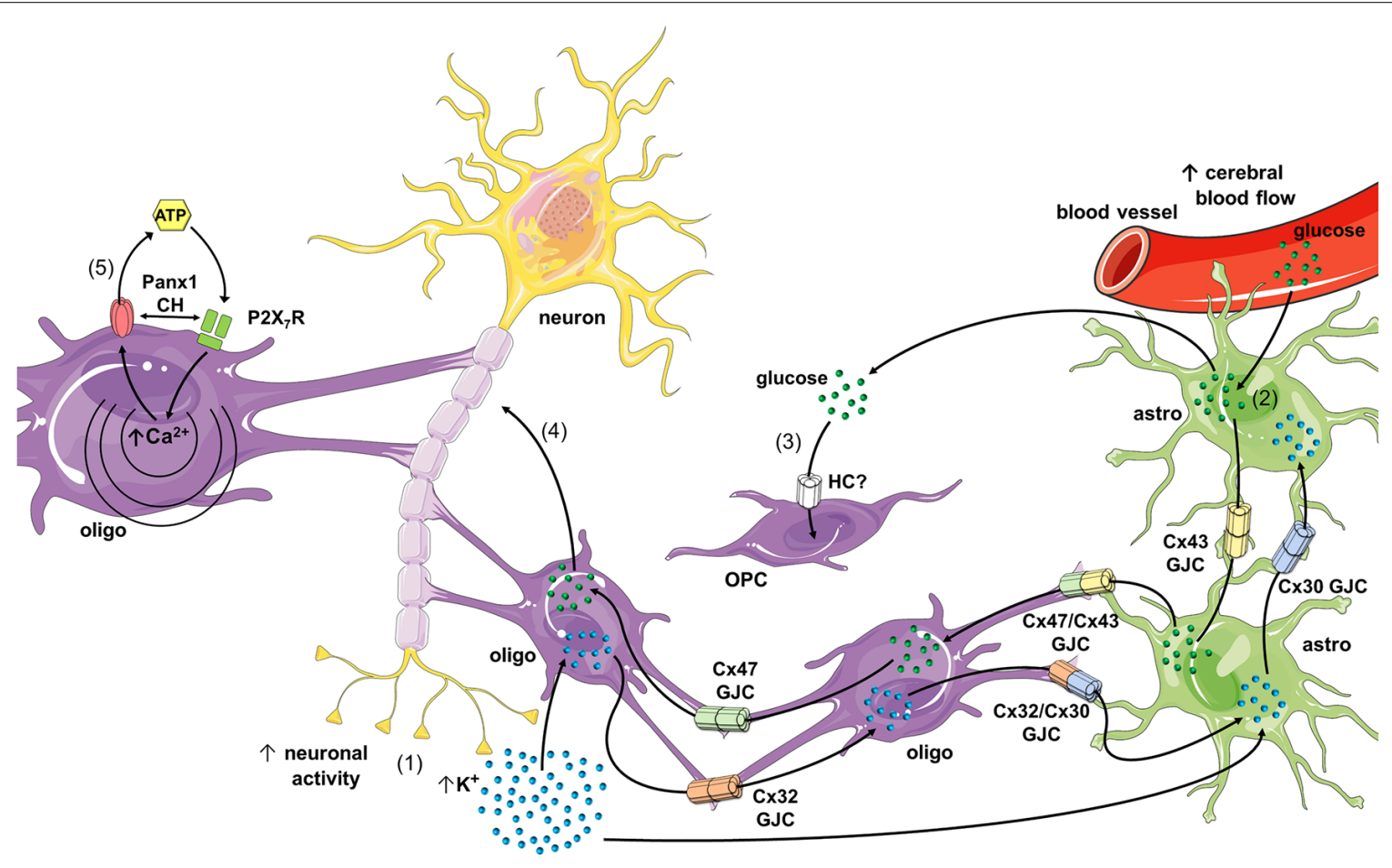

FIGURE 2 | Schematics of possible roles of connexin- and pannexin-based channels in oligodendrocyte function and dysfunction. During high rates of neuronal activity $\mathrm{K}^{+}$accumulates in the extracellular space, and then is taken up by oligodendrocytes and astrocytes through the inwardly rectifying $\mathrm{K}^{+}$channel (Kir) $4.1 \mathrm{and} / \mathrm{or} \mathrm{Na}^{+} / \mathrm{K}^{+}$-pumps (1). $\mathrm{K}^{+}$that concentrates inside oligodendrocytes and astrocytes diffuses to the panglial syncytium via homocellular and heterocellular gap junction channels (GJCs), a process termed "spatial $\mathrm{K}^{+}$buffering." In parallel, neuronal and astroglial signaling (e.g., ATP and glutamate) could activate endothelial P2 and NMDA receptors (NMDARs), respectively, leading to increased free [Ca $\left.{ }^{2+}\right]_{i}$ and further vasodilation of blood vessels (not depicted). The latter increases cerebral blood flow and further uptake of glucose by astrocytic endfeet (2). Glucose diffuses through astrocytes and oligodendrocytes via homocellular and heterocellular GJCs and then can be metabolized to lactate by astrocytes, and both can be released into the extracellular space. In addition, glucose is taken up by oligodendrocyte precursor cells (OPCs; via an unknown hemichannel) and neurons, which possibly modulate oligodendrocyte differentiation and maturation (3), as well as axonal function (4), respectively. In pathological scenarios, the opening of Panx1 channels may lead to the release of ATP from oligodendrocytes (5), resulting in the activation of $\mathrm{P} 2 \mathrm{X}_{7}$ receptors $\left(\mathrm{P} 2 \mathrm{X}_{7} \mathrm{Rs}\right)$ and further triggering of a self-perpetuating mechanism of cell damage, in which high levels of $\left[\text { Ca }{ }^{2+}\right]_{i}$ and direct protein-protein interaction could reactivate pannexons. Part of this schematics was done with support of the free online Servier Medical Art repository (https://smart.servier.com/). 
(e.g., microglia, mast cells or astrocytes) remain to be fully elucidated.

The functional expression of Panxl channels in oligodendrocytes was first described by Domercq et al. (2010). Using primary cultures of oligodendrocytes, they demonstrated that oxygen and glucose deprivation (OGD) increases ATP release and ischemic ionic imbalance-induced cell death by a mechanism involving the activation of $\mathrm{P}_{2} \mathrm{X}_{7} \mathrm{Rs}$ and Panx1 channels (Figure 2; Domercq et al., 2010). These findings were paralleled with increased intracellular $\mathrm{Ca}^{2+}$ concentration $\left(\left[\mathrm{Ca}^{2+}\right]_{\mathrm{i}}\right)$, which agrees with the fact that high extracellular ATP may trigger a rise of cytosolic $\mathrm{Ca}^{2+}$ in oligodendrocytes by activating P2XRs (James and Butt, 2001). $\mathrm{P}_{2} \mathrm{X}_{7} \mathrm{R}$ stimulation may lead to ATP release in oligodendrocytes through at least one mechanism implicating hemichannels and/or pannexons. Despite that $\mathrm{P} 2 \mathrm{X}_{7} \mathrm{R}$ activation rises $\left[\mathrm{Ca}^{2+}\right]_{\mathrm{i}}$ (Baroja-Mazo et al., 2013) and opens Cx43 hemichannels and Panx1 channels (Locovei et al., 2006; De Bock et al., 2012), the Panxl-mediated release of ATP depends on protein-protein interactions between $\mathrm{P}_{2} \mathrm{X}_{7} \mathrm{Rs}$ and Panx1 (Locovei et al., 2007). Consistent with this notion, Panx1 co-immunoprecipitates with $\mathrm{P}_{2} \mathrm{X}_{7} \mathrm{Rs}$ (Silverman et al., 2009; Poornima et al., 2012), where the proline 451 in the C-terminal tails of these receptors has been involved (Iglesias et al., 2008; Sorge et al., 2012). The positive feedback loop of activation among $\mathrm{P}_{2} \mathrm{X}_{7} \mathrm{Rs}$ and hemichannels/pannexons could explain the oligodendrocyte damage occurring in EAE, where high release of ATP has been linked to P2XR activation (Matute et al., 2007). Supporting this line of thought, probenecid, a Panx1 channel blocker, drastically prevent inflammation and oligodendrocyte damage triggered by cuprizone diet, a well-known model of demyelination (Hainz et al., 2017).

Further studies are necessary for elucidating the nature of the connexins involved in the activity of oligodendrocyte hemichannels, as well as how these channels and pannexons participate in the pathogenesis of diseases characterized by demyelination and oligodendrocyte dysfunction.

\section{CONCLUSIONS}

Oligodendrocytes serve to maintain critical brain functions such as myelination, metabolic supply, ion buffering, and recent findings suggest also a possible contribution to setting neuronal bursting properties. The evidence discussed in this review indicates that both oligodendrocyte-oligodendrocyte coupling (mainly mediated by $\mathrm{Cx} 47$ and $\mathrm{Cx} 32$ homotypic GJCs) and oligodendrocyte-astrocyte coupling (mediated by $\mathrm{Cx} 47 / \mathrm{Cx} 43$ or $\mathrm{Cx} 32 / \mathrm{Cx} 30$ pairs) play a pivotal role in forming a functional

\section{REFERENCES}

Abascal, F., and Zardoya, R. (2013). Evolutionary analyses of gap junction protein families. Biochim. Biophys. Acta 1828, 4-14. doi: 10.1016/j.bbamem.2012. 02.007

Ahn, M., Lee, J., Gustafsson, A., Enriquez, A., Lancaster, E., Sul, J. Y., et al. (2008). Cx29 and Cx32, two connexins expressed by myelinating glia, do not interact panglial syncytium supporting the buffering of extracellular $\mathrm{K}^{+}$and metabolic supply to axons. Although the available literature suggests that oligodendrocyte progenitors would not contribute to this functional syncytial network, their role could not be completely ruled out based on recent findings showing the coupling of OPCs with astrocytes through $\mathrm{Cx} 47$ (Liu et al., 2017; Xu et al., 2017). Future research will elucidate whether OPCs make part of this interconnected glial network. Of interest is the fact that both OPCs and mature oligodendrocytes are endowed with hemichannels that allow the passage of relevant bioactive molecules (e.g., glucose), which might be involved not only in oligodendroglia maturation, but also in metabolic coupling and energy supply to neurons.

The fact that patients with demyelinating diseases (i.e., MS) exhibits anomalies in normal expression and distribution of brain connexins, pointing out a specific role for hemichannels and GJCs in these neuropathologies. The studies reviewed here strength the recent body of evidence supporting a dysregulation of hemichannels and GJCs in glial cell types as a common phenomenon to several neurogenerative diseases. Future investigation should uncover the contribution of connexin- and pannexin-based channels to the onset and progression of demyelinating diseases.

\section{AUTHOR CONTRIBUTIONS}

JAO, MR and FCO conceived and designed the major ideas developed in the manuscript. JAO designed the Figure 2. All authors wrote and edited the manuscript. All authors read and approved the final manuscript.

\section{FUNDING}

This work was supported by Comisión Nacional de Investigación Científica y Tecnológica (CONICYT) and Programa de Investigación Asociativa (PIA): Grant Anillo de Ciencia y Tecnología ACT1411 (JAO); Fondo Nacional de Desarrollo Científico y Tecnológico (FONDECYT): Grant 1160710 (JAO), 11160616 (FCO) and 1160227 (MR) and Programa de Cooperación Científica ECOS-CONICYT C18S02 (MR, FCO and JAO).

\section{ACKNOWLEDGMENTS}

We thank CONICYT, PIA, PCI, FONDECYT, Clínica Alemana Universidad del Desarrollo, Universidad Autónoma de Chile and Pontificia Universidad Católica de Chile.

and are functionally distinct. J. Neurosci. Res. 86, 992-1006. doi: 10.1002/jnr. 21561

Altevogt, B. M., and Paul, D. L. (2004). Four classes of intercellular channels between glial cells in the CNS. J. Neurosci. 24, 4313-4323. doi: 10.1523/JNEUROSCI.3303-03.2004

Altevogt, B. M., Kleopa, K. A., Postma, F. R., Scherer, S. S., and Paul, D. L. (2002). Connexin29 is uniquely distributed within myelinating glial cells 
of the central and peripheral nervous systems. J. Neurosci. 22, 6458-6470. doi: 10.1523/JNEUROSCI.22-15-06458.2002

Araque, A., Carmignoto, G., Haydon, P. G., Oliet, S. H., Robitaille, R., and Volterra, A. (2014). Gliotransmitters travel in time and space. Neuron 81, 728-739. doi: 10.1016/j.neuron.2014. 02.007

Balice-Gordon, R. J., Bone, L. J., and Scherer, S. S. (1998). Functional gap junctions in the schwann cell myelin sheath. J. Cell Biol. 142, 1095-1104. doi: 10.1083/jcb. 142.4.1095

Baroja-Mazo, A., Barberà-Cremades, M., and Pelegrin, P. (2013). The participation of plasma membrane hemichannels to purinergic signaling. Biochim. Biophys. Acta 1828, 79-93. doi: 10.1016/j.bbamem.2012.01.002

Battefeld, A., Klooster, J., and Kole, M. H. (2016). Myelinating satellite oligodendrocytes are integrated in a glial syncytium constraining neuronal high-frequency activity. Nat. Commun. 7:11298. doi: 10.1038/ncomms 11298

Bergles, D. E., Roberts, J. D., Somogyi, P., and Jahr, C. E. (2000). Glutamatergic synapses on oligodendrocyte precursor cells in the hippocampus. Nature 405, 187-191. doi: 10.1038/35012083

Bruzzone, R., Hormuzdi, S. G., Barbe, M. T., Herb, A., and Monyer, H. (2003). Pannexins, a family of gap junction proteins expressed in brain. Proc. Natl. Acad. Sci. U S A 100, 13644-13649. doi: 10.1073/pnas.2233464100

Chever, O., Lee, C. Y., and Rouach, N. (2014). Astroglial connexin 43 hemichannels tune basal excitatory synaptic transmission. J. Neurosci. 34, 11228-11232. doi: 10.1523/JNEUROSCI.0015-14.2014

Constantinescu, C., Farooqi, N., O’Brien, K., and Gran, B. (2011). Experimental autoimmune encephalomyelitis (EAE) as a model for multiple sclerosis (MS). Br. J. Pharmacol. 164, 1079-1106. doi: 10.1111/j.1476-5381.2011.01302.x

Cotrina, M. L., and Nedergaard, M. (2012). Brain connexins in demyelinating diseases: therapeutic potential of glial targets. Brain Res. 1487, 61-68. doi: 10.1016/j.brainres.2012.07.003

De Bock, M., Wang, N., Bol, M., Decrock, E., Ponsaerts, R., Bultynck, G., et al. (2012). Connexin 43 hemichannels contribute to cytoplasmic $\mathrm{Ca}^{2+}$ oscillations by providing a bimodal $\mathrm{Ca}^{2+}$-dependent $\mathrm{Ca}^{2+}$ entry pathway. J. Biol. Chem. 287, 12250-12266. doi: 10.1074/jbc.M111.299610

del Río-Hortega, P. (1922). ?'Son homologables la glía de escasas radiaciones y la celula de Schwann. Bol. Soc. Esp. Biol. 10, 25-28.

Dermietzel, R., Traub, O., Hwang, T. K., Beyer, E., Bennett, M. V., Spray, D. C., et al. (1989). Differential expression of three gap junction proteins in developing and mature brain tissues. Proc. Natl. Acad. Sci. U S A 86, 10148-10152. doi: 10.1073/pnas.86.24.10148

Dimou, L., and Simons, M. (2017). Diversity of oligodendrocytes and their progenitors. Curr. Opin. Neurobiol. 47, 73-79. doi: 10.1016/j.conb.2017.09.015

Domercq, M., Perez-Samartin, A., Aparicio, D., Alberdi, E., Pampliega, O., and Matute, C. (2010). P2X 7 receptors mediate ischemic damage to oligodendrocytes. Glia 58, 730-740. doi: 10.1002/glia.20958

Fairweather, N., Bell, C., Cochrane, S., Chelly, J., Wang, S., Mostacciuolo, M. L., et al. (1994). Mutations in the connexin 32 gene in X-linked dominant Charcot-Marie-Tooth disease (CMTX1). Hum. Mol. Genet 3, 1033-1034. doi: $10.1093 / \mathrm{hmg} / 3.6 .1033-\mathrm{a}$

Fasciani, I., Pluta, P., González-Nieto, D., Martínez-Montero, P., Molano, J., Paino, C. L., et al. (2018). Directional coupling of oligodendrocyte connexin-47 and astrocyte connexin-43 gap junctions. Glia 66, 2340-2352. doi: 10.1002/glia. 23471

Fields, R. D., Dutta, D. J., Belgrad, J., and Robnett, M. (2017). Cholinergic signaling in myelination. Glia 65, 687-698. doi: 10.1002/glia.23101

Flagg-Newton, J. L., and Loewenstein, W. R. (1980). Asymmetrically permeable membrane channels in cell junction. Science 207, 771-773. doi: 10.1126/science. 7352287

Franklin, R. J., and Ffrench-Constant, C. (2008). Remyelination in the CNS: from biology to therapy. Nat. Rev. Neurosci. 9, 839-855. doi: 10.1038/nrn2480

Franklin, R. J. M., and Ffrench-Constant, C. (2017). Regenerating CNS myelin-from mechanisms to experimental medicines. Nat. Rev. Neurosci. 18, 753-769. doi: 10.1038/nrn.2017.136

Fulmer, C. G., VonDran, M. W., Stillman, A. A., Huang, Y., Hempstead, B. L., and Dreyfus, C. F. (2014). Astrocyte-derived BDNF supports myelin protein synthesis after cuprizone-induced demyelination. J. Neurosci. 34, 8186-8196. doi: 10.1523/JNEUROSCI.4267-13.2014
Giaume, C., Fromaget, C., El Aoumari, A., Cordier, J., Glowinski, J., and Gros, D. (1991). Gap junctions in cultured astrocytes: single-channel currents and characterization of channel-forming protein. Neuron 6, 133-143. doi: 10.1016/0896-6273(91)90128-m

Gonatas, N. K., Hirayama, M., Stieber, A., and Silberberg, D. H. (1982). The ultrastructure of isolated rat oligodendroglial cell cultures. J. Neurocytol. 11, 997-1008. doi: 10.1007/bf01148313

Hainz, N., Becker, P., Rapp, D., Wagenpfeil, S., Wonnenberg, B., Beisswenger, C., et al. (2017). Probenecid-treatment reduces demyelination induced by cuprizone feeding. J. Chem. Neuroanat. 85, 21-26. doi: 10.1016/j.jchemneu. 2017.06.003

Hamada, M. S., and Kole, M. H. (2015). Myelin loss and axonal ion channel adaptations associated with gray matter neuronal hyperexcitability. J. Neurosci. 35, 7272-7286. doi: 10.1523/JNEUROSCI.4747-14.2015

Hammond, T. R., McEllin, B., Morton, P. D., Raymond, M., Dupree, J., and Gallo, V. (2015). Endothelin-B receptor activation in astrocytes regulates the rate of oligodendrocyte regeneration during remyelination. Cell Rep. 13, 2090-2097. doi: 10.1016/j.celrep.2015.11.002

Iglesias, R., Locovei, S., Roque, A., Alberto, A. P., Dahl, G., Spray, D. C., et al. (2008). P2 $\mathrm{X}_{7}$ receptor-Pannexin1 complex: pharmacology and signaling. Am. J. Physiol. Cell Physiol. 295, C752-C760. doi: 10.1152/ajpcell.00228.2008

Ishibashi, T., Dakin, K. A., Stevens, B., Lee, P. R., Kozlov, S. V., Stewart, C. L., et al. (2006). Astrocytes promote myelination in response to electrical impulses. Neuron 49, 823-832. doi: 10.1016/j.neuron.2006.02.006

James, G., and Butt, A. M. (2001). P2X and P2Y purinoreceptors mediate ATP-evoked calcium signalling in optic nerve glia in situ. Cell Calcium 30, 251-259. doi: 10.1054/ceca.2001.0232

Kamasawa, N., Sik, A., Morita, M., Yasumura, T., Davidson, K. G., Nagy, J. I., et al. (2005). Connexin-47 and connexin-32 in gap junctions of oligodendrocyte somata, myelin sheaths, paranodal loops and Schmidt-Lanterman incisures: implications for ionic homeostasis and potassium siphoning. Neuroscience 136, 65-86. doi: 10.1016/j.neuroscience.2005.08.027

Kettenmann, H., Orkand, R. K., and Schachner, M. (1983). Coupling among identified cells in mammalian nervous system cultures. J. Neurosci. 3, 506-516. doi: 10.1523/jneurosci.03-03-00506.1983

Kleopa, K. A., Orthmann, J. L., Enriquez, A., Paul, D. L., and Scherer, S. S. (2004). Unique distributions of the gap junction proteins connexin29, connexin 32 and connexin 47 in oligodendrocytes. Glia 47, 346-357. doi: 10.1002/glia.20043

Kleopa, K. A., Orthmann-Murphy, J., and Sargiannidou, I. (2010). Gap junction disorders of myelinating cells. Rev. Neurosci. 21, 397-419. doi: 10.1515/revneuro.2010.21.5.397

Kleopa, K. A., Yum, S. W., and Scherer, S. S. (2002). Cellular mechanisms of connexin 32 mutations associated with CNS manifestations. J. Neurosci. Res. 68 , 522-534. doi: 10.1002/jnr.10255

Kukley, M., Capetillo-Zarate, E., and Dietrich, D. (2007). Vesicular glutamate release from axons in white matter. Nat. Neurosci. 10, 311-320. doi: $10.1038 / \mathrm{nn} 1850$

Kunzelmann, P., Blümcke, I., Traub, O., Dermietzel, R., and Willecke, K. (1997). Coexpression of connexin 45 and -32 in oligodendrocytes of rat brain. J. Neurocytol. 26, 17-22. doi: 10.1023/A:1018555207379

Leybaert, L., Lampe, P. D., Dhein, S., Kwak, B. R., Ferdinandy, P., Beyer, E. C., et al. (2017). Connexins in cardiovascular and neurovascular health and disease: pharmacological implications. Pharmacol. Rev. 69, 396-478. doi: 10.1124/pr. 115.012062

Li, X., Ionescu, A. V., Lynn, B. D., Lu, S., Kamasawa, N., Morita, M., et al. (2004). Connexin47, connexin29 and connexin32 co-expression in oligodendrocytes and Cx47 association with zonula occludens-1 (ZO-1) in mouse brain. Neuroscience 126, 611-630. doi: 10.1016/j.neuroscience.2004. 03.063

Li, C., Xiao, L., Liu, X., Yang, W., Shen, W., Hu, C., et al. (2013). A functional role of NMDA receptor in regulating the differentiation of oligodendrocyte precursor cells and remyelination. Glia 61, 732-749. doi: 10.1002/glia.22469

Lin, S. C., and Bergles, D. E. (2004). Synaptic signaling between GABAergic interneurons and oligodendrocyte precursor cells in the hippocampus. Nat. Neurosci. 7, 24-32. doi: 10.1038/nn1162

Lin, J. H., Lou, N., Kang, N., Takano, T., Hu, F., Han, X., et al. (2008). A central role of connexin 43 in hypoxic preconditioning. J. Neurosci. 28, 681-695. doi: 10.1523/JNEUROSCI.3827-07.2008 
Liu, Z., Xu, D., Wang, S., Chen, Y., Li, Z., Gao, X., et al. (2017). Astrocytes induce proliferation of oligodendrocyte progenitor cells via connexin 47-mediated activation of the ERK/Id4 pathway. Cell Cycle 16, 714-722. doi: 10.1080/15384101.2017.1295183

Locovei, S., Scemes, E., Qiu, F., Spray, D. C., and Dahl, G. (2007). Pannexin1 is part of the pore forming unit of the $\mathrm{P}_{2} \mathrm{X}_{7}$ receptor death complex. FEBS Lett. 581, 483-488. doi: 10.1016/j.febslet.2006.12.056

Locovei, S., Wang, J., and Dahl, G. (2006). Activation of pannexin 1 channels by ATP through P2Y receptors and by cytoplasmic calcium. FEBS Lett. 580, 239-244. doi: 10.1016/j.febslet.2005.12.004

Ludwin, S. K. (1979). The perineuronal satellite oligodendrocyte. A role in remyelination. Acta Neuropathol. 47, 49-53. doi: 10.1007/bf00698272

Maglione, M., Tress, O., Haas, B., Karram, K., Trotter, J., Willecke, K., et al. (2010). Oligodendrocytes in mouse corpus callosum are coupled via gap junction channels formed by connexin 47 and connexin32. Glia 58, 1104-1117. doi: 10.1002/glia.20991

Magnotti, L. M., Goodenough, D. A., and Paul, D. L. (2011). Functional heterotypic interactions between astrocyte and oligodendrocyte connexins. Glia 59, 26-34. doi: 10.1002/glia.21073

Markoullis, K., Sargiannidou, I., Gardner, C., Hadjisavvas, A., Reynolds, R., and Kleopa, K. A. (2012). Disruption of oligodendrocyte gap junctions in experimental autoimmune encephalomyelitis. Glia 60, 1053-1066. doi: 10.1002/glia.22334

Markoullis, K., Sargiannidou, I., Schiza, N., Roncaroli, F., Reynolds, R., and Kleopa, K. A. (2014). Oligodendrocyte gap junction loss and disconnection from reactive astrocytes in multiple sclerosis gray matter. J. Neuropathol. Exp. Neurol. 73, 865-879. doi: 10.1097/NEN.0000000000000106

Massa, P. T., and Mugnaini, E. (1982). Cell junctions and intramembrane particles of astrocytes and oligodendrocytes: a freeze-fracture study. Neuroscience 7, 523-538. doi: 10.1016/0306-4522(82)90285-8

Massa, P. T., Szuchet, S., and Mugnaini, E. (1984). Cell-cell interactions of isolated and cultured oligodendrocytes: formation of linear occluding junctions and expression of peculiar intramembrane particles. J. Neurosci. 4, 3128-3139. doi: 10.1523/jneurosci.04-12-03128.1984

Maturana, C. J., Aguirre, A., and Saez, J. C. (2017). High glucocorticoid levels during gestation activate the inflammasome in hippocampal oligodendrocytes of the offspring. Dev. Neurobiol. 77, 625-642. doi: 10.1002/dneu.22409

Matute, C., Torre, I., Pérez-Cerdá, F., Pérez-Samartín, A., Alberdi, E., Etxebarria, E., et al. (2007). P2X 7 receptor blockade prevents ATP excitotoxicity in oligodendrocytes and ameliorates experimental autoimmune encephalomyelitis. J. Neurosci. 27, 9525-9533. doi: 10.1523/JNEUROSCI.057907.2007

May, D., Tress, O., Seifert, G., and Willecke, K. (2013). Connexin47 protein phosphorylation and stability in oligodendrocytes depend on expression of Connexin43 protein in astrocytes. J. Neurosci. 33, 7985-7996. doi: 10.1523/JNEUROSCI.5874-12.2013

Menichella, D. M., Majdan, M., Awatramani, R., Goodenough, D. A., Sirkowski, E., Scherer, S. S., et al. (2006). Genetic and physiological evidence that oligodendrocyte gap junctions contribute to spatial buffering of potassium released during neuronal activity. J. Neurosci. 26, 10984-10991. doi: 10.1523/JNEUROSCI.0304-06.2006

Meunier, C., Wang, N., Yi, C., Dallerac, G., Ezan, P., Koulakoff, A., et al. (2017). Contribution of astroglial Cx43 hemichannels to the modulation of glutamatergic currents by D-serine in the mouse prefrontal cortex. J. Neurosci. 37, 9064-9075. doi: 10.1523/JNEUROSCI.2204-16.2017

Meyer, N., Richter, N., Fan, Z., Siemonsmeier, G., Pivneva, T., Jordan, P., et al. (2018). Oligodendrocytes in the mouse corpus callosum maintain axonal function by delivery of glucose. Cell Rep. 22, 2383-2394. doi: 10.1016/j.celrep. 2018.02.022

Micevych, P. E., and Abelson, L. (1991). Distribution of mRNAs coding for liver and heart gap junction proteins in the rat central nervous system. J. Comp. Neurol. 305, 96-118. doi: 10.1002/cne.903050110

Miron, V. E., Boyd, A., Zhao, J. W., Yuen, T. J., Ruckh, J. M., Shadrach, J. L., et al. (2013). M2 microglia and macrophages drive oligodendrocyte differentiation during CNS remyelination. Nat. Neurosci. 16, 1211-1218. doi: 10.1038/ nn.3469

Miyamoto, N., Maki, T., Shindo, A., Liang, A. C., Maeda, M., Egawa, N., et al. (2015). Astrocytes promote oligodendrogenesis after white matter damage via brain-derived neurotrophic factor. J. Neurosci. 35, 14002-14008. doi: 10.1523/JNEUROSCI.1592-15.2015

Montero, T. D., and Orellana, J. A. (2015). Hemichannels: new pathways for gliotransmitter release. Neuroscience 286, 45-59. doi: 10.1016/j.neuroscience. 2014.11.048

Nagy, J. I., Ionescu, A. V., Lynn, B. D., and Rash, J. E. (2003). Connexin29 and connexin 32 at oligodendrocyte and astrocyte gap junctions and in myelin of the mouse central nervous system. J. Comp. Neurol. 464, 356-370. doi: 10.1002/cne. 10797

Nagy, J. I., Li, X., Rempel, J., Stelmack, G., Patel, D., Staines, W. A., et al. (2001). Connexin 26 in adult rodent central nervous system: demonstration at astrocytic gap junctions and colocalization with connexin 30 and connexin 43 . J. Comp. Neurol. 441, 302-323. doi: 10.1002/cne.1414

Nave, K. A., and Werner, H. B. (2014). Myelination of the nervous system: mechanisms and functions. Annu. Rev. Cell Dev. Biol. 30, 503-533. doi: 10.1146/annurev-cellbio-100913-013101

Nelis, E., Warner, L. E., Vriendt, E. D., Chance, P. F., Lupski, J. R., and Van Broeckhoven, C. (1996). Comparison of single-strand conformation polymorphism and heteroduplex analysis for detection of mutations in Charcot-Marie-Tooth type 1 disease and related peripheral neuropathies. Eur. J. Hum. Genet. 4, 329-333. doi: 10.1159/000472227

Niu, J., Li, T., Yi, C., Huang, N., Koulakoff, A., Weng, C., et al. (2016). Connexinbased channels contribute to metabolic pathways in the oligodendroglial lineage. J. Cell Sci. 129, 1902-1914. doi: 10.1242/jcs.178731

Norton, W. T., Farooq, M., Fields, K. L., and Raine, C. S. (1983). The long term culture of bulk-isolated bovine oligodendroglia from adult brain. Brain Res. 270, 295-310. doi: 10.1016/0006-8993(83)90604-2

Nualart-Marti, A., Solsona, C., and Fields, R. D. (2013). Gap junction communication in myelinating glia. Biochim. Biophys. Acta 1828, 69-78. doi: 10.1016/j.bbamem.2012.01.024

Olympiou, M., Sargiannidou, I., Markoullis, K., Karaiskos, C., Kagiava, A., Kyriakoudi, S., et al. (2016). Systemic inflammation disrupts oligodendrocyte gap junctions and induces ER stress in a model of CNS manifestations of X-linked Charcot-Marie-Tooth disease. Acta Neuropathol. Commun. 4:95. doi: 10.1186/s40478-016-0369-5

Orduz, D., Maldonado, P. P., Balia, M., Vélez-Fort, M., de Sars, V., Yanagawa, Y., et al. (2015). Interneurons and oligodendrocyte progenitors form a structured synaptic network in the developing neocortex. Elife 4:e06953. doi: 10.7554/elife. 06953

Orellana, J. A., Retamal, M. A., Moraga-Amaro, R., and Stehberg, J. (2016). Role of astroglial hemichannels and pannexons in memory and neurodegenerative diseases. Front. Integr. Neurosci. 10:26. doi: 10.3389/fnint.2016.00026

Orellana, J. A., Sáez, P. J., Cortés-Campos, C., Elizondo, R. J., Shoji, K. F., Contreras-Duarte, S., et al. (2012). Glucose increases intracellular free $\mathrm{Ca}^{2+}$ in tanycytes via ATP released through connexin 43 hemichannels. Glia 60, 53-68. doi: 10.1002 /glia. 21246

Orthmann-Murphy, J. L., Abrams, C. K., and Scherer, S. S. (2008). Gap junctions couple astrocytes and oligodendrocytes. J. Mol. Neurosci. 35, 101-116. doi: 10.1007/s12031-007-9027-5

Orthmann-Murphy, J. L., Enriquez, A. D., Abrams, C. K., and Scherer, S. S. (2007a). Loss-of-function GJA12/Connexin47 mutations cause PelizaeusMerzbacher-like disease. Mol. Cell. Neurosci. 34, 629-641. doi: 10.1016/j.mcn. 2007.01.010

Orthmann-Murphy, J. L., Freidin, M., Fischer, E., Scherer, S. S., and Abrams, C. K. (2007b). Two distinct heterotypic channels mediate gap junction coupling between astrocyte and oligodendrocyte connexins. J. Neurosci. 27, 13949-13957. doi: 10.1523/JNEUROSCI.3395-07.2007

Osaka, H., Hamanoue, H., Yamamoto, R., Nezu, A., Sasaki, M., Saitsu, H., et al. (2010). Disrupted SOX10 regulation of GJC2 transcription causes PelizaeusMerzbacher-like disease. Ann. Neurol. 68, 250-254. doi: 10.1002/ana.22022

Pastor, A., Kremer, M., Möller, T., Kettenmann, H., and Dermietzel, R. (1998). Dye coupling between spinal cord oligodendrocytes: differences in coupling efficiency between gray and white matter. Glia 24, 108-120. doi: 10.1002/(sici)1098-1136(199809)24:1<108::aid-glia11>3.0.co;2-v

Paul, D. L., Ebihara, L., Takemoto, L. J., Swenson, K. I., and Goodenough, D. A. (1991). Connexin46, a novel lens gap junction protein, induces voltage-gated currents in nonjunctional plasma membrane of Xenopus oocytes J. Cell Biol. 115, 1077-1089. doi: 10.1083/jcb.115.4.1077 
Pérez-Cerdá, F., Sánchez-Gómez, M. V., and Matute, C. (2015). Pío del Río Hortega and the discovery of the oligodendrocytes. Front. Neuroanat. 9:92. doi: $10.3389 /$ fnana.2015.00092

Philips, T., and Rothstein, J. D. (2017). Oligodendroglia: metabolic supporters of neurons. J. Clin. Invest. 127, 3271-3280. doi: 10.1172/jci90610

Poornima, V., Madhupriya, M., Kootar, S., Sujatha, G., Kumar, A., and Bera, A. K. (2012). P2X 7 receptor-pannexin 1 hemichannel association: effect of extracellular calcium on membrane permeabilization. J. Mol. Neurosci. 46, 585-594. doi: 10.1007/s12031-011-9646-8

Pozniak, C. D., Langseth, A. J., Dijkgraaf, G. J., Choe, Y., Werb, Z., and Pleasure, S. J. (2010). Sox10 directs neural stem cells toward the oligodendrocyte lineage by decreasing Suppressor of Fused expression. Proc. Natl. Acad. Sci. U S A 107, 21795-21800. doi: 10.1073/pnas.1016485107

Ramón y Cajal, S. (1925). "Notas técnicas," in Boletín de la Sociedad Española de Biología (Madrid: Sociedad española de biología) 25-28.

Rash, J. E. (2010). Molecular disruptions of the panglial syncytium block potassium siphoning and axonal saltatory conduction: pertinence to neuromyelitis optica and other demyelinating diseases of the central nervous system. Neuroscience 168, 982-1008. doi: 10.1016/j.neuroscience.2009.10.028

Rash, J. E., Vanderpool, K. G., Yasumura, T., Hickman, J., Beatty, J. T., and Nagy, J. I. (2016). KV1 channels identified in rodent myelinated axons, linked to Cx29 in innermost myelin: support for electrically active myelin in mammalian saltatory conduction. J. Neurophysiol. 115, 1836-1859. doi: 10.1152/jn.01077.2015

Roux, L., Madar, A., Lacroix, M. M., Yi, C., Benchenane, K., and Giaume, C. (2015). Astroglial connexin 43 hemichannels modulate olfactory bulb slow oscillations. J. Neurosci. 35, 15339-15352. doi: 10.1523/JNEUROSCI.0861-15.2015

Saab, A. S., Tzvetavona, I. D., Trevisiol, A., Baltan, S., Dibaj, P., Kusch, K., et al. (2016). Oligodendroglial NMDA receptors regulate glucose import and axonal energy metabolism. Neuron 91, 119-132. doi: 10.1016/j.neuron.2016. 05.016

Saez, J. C., Berthoud, V. M., Branes, M. C., Martinez, A. D., and Beyer, E. C. (2003). Plasma membrane channels formed by connexins: their regulation and functions. Physiol. Rev. 83, 1359-1400. doi: 10.1152/physrev.00007.2003

Sargiannidou, I., Ahn, M., Enriquez, A. D., Peinado, A., Reynolds, R., Abrams, C., et al. (2008). Human oligodendrocytes express Cx31.3: function and interactions with Cx32 mutants. Neurobiol. Dis. 30, 221-233. doi: 10.1016/j. nbd.2008.01.009

Sargiannidou, I., Markoullis, K., and Kleopa, K. A. (2010). Molecular mechanisms of gap junction mutations in myelinating cells. Hist. Histopathol. 25, 1191-1206. doi: 10.14670/HH-25.1191

Scherer, S. S., Xu, Y. T., Nelles, E., Fischbeck, K., Willecke, K., and Bone, L. J. (1998). Connexin32-null mice develop demyelinating peripheral neuropathy. Glia 24, 8-20. doi: 10.1002/(sici)1098-1136(199809)24:1<8::aid-glia2>3.0. co;2-3

Schlierf, B., Werner, T., Glaser, G., and Wegner, M. (2006). Expression of connexin47 in oligodendrocytes is regulated by the Sox 10 transcription factor. J. Mol. Biol. 361, 11-21. doi: 10.1016/j.jmb.2006.05.072

Silverman, W. R., de Rivero Vaccari, J. P., Locovei, S., Qiu, F., Carlsson, S. K., Scemes, E., et al. (2009). The pannexin 1 channel activates the inflammasome in neurons and astrocytes. J. Biol. Chem. 284, 18143-18151. doi: 10.1074/jbc. m109.004804

Sorge, R. E., Trang, T., Dorfman, R., Smith, S. B., Beggs, S., Ritchie, J., et al. (2012). Genetically determined $\mathrm{P}_{2} \mathrm{X}_{7}$ receptor pore formation regulates variability in chronic pain sensitivity. Nat. Med. 18, 595-599. doi: 10.1038/nm.2710

Sosinsky, G. E., Boassa, D., Dermietzel, R., Duffy, H. S., Laird, D. W., MacVicar, B., et al. (2011). Pannexin channels are not gap junction hemichannels. Channels 5, 193-197. doi: 10.4161/chan.5.3.15765

Stehberg, J., Moraga-Amaro, R., Salazar, C., Becerra, A., Echeverria, C., Orellana, J. A., et al. (2012). Release of gliotransmitters through astroglial connexin 43 hemichannels is necessary for fear memory consolidation in the basolateral amygdala. FASEB J. 26, 3649-3657. doi: 10.1096/fj.11-198416

Sutor, B., Schmolke, C., Teubner, B., Schirmer, C., and Willecke, K. (2000). Myelination defects and neuronal hyperexcitability in the neocortex of connexin 32-deficient mice. Cereb. Cortex 10, 684-697. doi: 10.1093/cercor/10. 7.684
Suzuki, N., Sekimoto, K., Hayashi, C., Mabuchi, Y., Nakamura, T., and Akazawa, C. (2017). Differentiation of oligodendrocyte precursor cells from Sox10-venus mice to oligodendrocytes and astrocytes. Sci. Rep. 7:14133. doi: 10.1038/s41598-017-14207-0

Szuchet, S., Nielsen, J. A., Lovas, G., Domowicz, M. S., de Velasco, J. M., Maric, D., et al. (2011). The genetic signature of perineuronal oligodendrocytes reveals their unique phenotype. Eur. J. Neurosci. 34, 1906-1922. doi: 10.1111/j.14609568.2011.07922.x

Takada, N., Kucenas, S., and Appel, B. (2010). Sox10 is necessary for oligodendrocyte survival following axon wrapping. Glia 58, 996-1006. doi: 10.1002/glia.20981

Takasaki, C., Yamasaki, M., Uchigashima, M., Konno, K., Yanagawa, Y., and Watanabe, M. (2010). Cytochemical and cytological properties of perineuronal oligodendrocytes in the mouse cortex. Eur. J. Neurosci. 32, 1326-1336. doi: 10.1111/j.1460-9568.2010.07377.x

Tasaki, I. (1939). The electro-saltatory transmission of the nerve impulse and the effect of narcosis upon the nerve fiber. Am. J. Physiol. Leg. Content127 211-227. doi: 10.1152/ajplegacy.1939.127.2.211

Tress, O., Maglione, M., May, D., Pivneva, T., Richter, N., Seyfarth, J., et al. (2012). Panglial gap junctional communication is essential for maintenance of myelin in the CNS. J. Neurosci. 32, 7499-7518. doi: 10.1523/JNEUROSCI.0392-12.2012

Tress, O., Maglione, M., Zlomuzica, A., May, D., Dicke, N., Degen, J., et al. (2011). Pathologic and phenotypic alterations in a mouse expressing a connexin47 missense mutation that causes Pelizaeus-Merzbacher-like disease in humans. PLoS Genet. 7:e1002146. doi: 10.1371/journal.pgen.1002146

Vélez-Fort, M., Audinat, E., and Angulo, M. C. (2009). Functional $\alpha$ 7-containing nicotinic receptors of NG2-expressing cells in the hippocampus. Glia 57, 1104-1114. doi: 10.1002/glia.20834

Von Blankenfeld, G., Ransom, B. R., and Kettenmann, H. (1993). Development of cell-cell coupling among cells of the oligodendrocyte lineage. Glia 7, 322-328. doi: 10.1002/glia.440070407

Wake, H., Ortiz, F. C., Woo, D. H., Lee, P. R., Angulo, M. C., and Fields, R. D. (2015). Nonsynaptic junctions on myelinating glia promote preferential myelination of electrically active axons. Nat. Commun. 6:7844. doi: $10.1038 /$ ncomms 8844

Walz, W. (2000). Role of astrocytes in the clearance of excess extracellular potassium. Neurochem. Int. 36, 291-300. doi: 10.1016/s0197-0186(99)00137-0

Wasseff, S. K., and Scherer, S. S. (2011). Cx32 and Cx47 mediate oligodendrocyte:astrocyte and oligodendrocyte:oligodendrocyte gap junction coupling. Neurobiol. Dis. 42, 506-513. doi: 10.1016/j.nbd.2011.03.003

Wasseff, S. K., and Scherer, S. S. (2015). Activated immune response in an inherited leukodystrophy disease caused by the loss of oligodendrocyte gap junctions. Neurobiol. Dis. 82, 86-98. doi: 10.1016/j.nbd.2015.05.018

Xu, D., Liu, Z., Wang, S., Peng, Y., and Sun, X. (2017). Astrocytes regulate the expression of Sp1R3 on oligodendrocyte progenitor cells through Cx47 and promote their proliferation. Biochem. Biophys. Res. Commun. 490, 670-675. doi: 10.1016/j.bbrc.2017.06.099

$\mathrm{Xu}$, G., Wang, W., and Zhou, M. (2014). Spatial organization of NG2 glial cells and astrocytes in rat hippocampal CA1 region. Hippocampus 24, 383-395. doi: 10.1002/hipo. 22232

Zlomuzica, A., Tress, O., Binder, S., Rovira, C., Willecke, K., and Dere, E. (2012). Changes in object recognition and anxiety-like behaviour in mice expressing a Cx47 mutation that causes Pelizaeus-Merzbacher-like disease. Dev. Neurosci. 34, 277-287. doi: 10.1159/000339305

Conflict of Interest Statement: The authors declare that the research was conducted in the absence of any commercial or financial relationships that could be construed as a potential conflict of interest.

Copyright (c) 2019 Vejar, Oyarzún, Retamal, Ortiz and Orellana. This is an open-access article distributed under the terms of the Creative Commons Attribution License (CC BY). The use, distribution or reproduction in other forums is permitted, provided the original author(s) and the copyright owner(s) are credited and that the original publication in this journal is cited, in accordance with accepted academic practice. No use, distribution or reproduction is permitted which does not comply with these terms. 